\title{
Einige Bemerkungen zur Wissensgeschichte der Materien
}

Bettina Wahrig

\begin{abstract}
Ursula Klein und Emma C. Spary (Hg.) 2010: Materials and Expertise in Early Modern Europe. Between Market and Laboratory. Chicago, London: University of Chicago Press, geb., 408 S., 50,00 \$, ISBN-13: 978-0-2264-3968-6.
\end{abstract}

Pratik Chakrabarti 2010: Materials and Medicine. Trade, Conquest and Therapeutics in the Eighteenth Century. [= Studies in Imperialism] Manchester: Manchester University Press, geb., $259 \mathrm{~S}$., 60,00 f, ISBN-13: 978-0-7190-8312-9.

Seit dem practical turn umkreisen die Gedanken der Wissenshistorikerinnen und Wissenshistoriker ${ }^{1}$ das Problem des Materiellen wie Goethes Falter die Flamme. Egal, ob Wissenschaft aus der Perspektive von "Science in the making", als Assemblage von menschlichen und nichtmenschlichen Akteuren, als Teil eines kulturellen Settings oder noch anders verstanden wird, immer scheint die Abkehr von einem traditionell theoriegeleiteten Wissenschaftsverständnis es notwendig zu machen, nicht nur über die geänderte Rolle der Theorie, nicht nur über eine andere Auffassung des Theorie-Praxis-Verhältnisses nachzudenken, sondern auch darüber, was das gegenüber der Theorie aufgewertete Materielle denn ist. Das fängt schon mit den Benennungen an: Nicht mehr Materie, sondern Materien oder Material interessieren uns. Ein paralleler Impuls kam aus den Kulturwissenschaften, die mit dem Schlagwort der "materiellen Kultur" ${ }^{2}$ eine Reihe von Interpretationsmustern entwickelten. Stellvertretend für die Vielzahl der neueren Ansätze seien hier nur die von Christina von Braun betriebene Kulturgeschichte in der longue durée, zuletzt 2012 zur Kulturgeschichte des Geldes, sowie die sich auf eine symmetrische Ontologie berufenden Arbeiten Bruno Latours, etwa Die Hoffnung der Pandora, genannt. Bei dem von Michael Werner und Bénédicte Zimmermann 
vorgeschlagenen Ansatz der Histoire croisée scheint mir besonders interessant, dass Intersektionen auf Mikro- oder Makroebene, aber auch auf verschiedenen geographischen oder Zeitskalen betrachtet werden können. Damit verliert das bisherige Objekt der Forschung seine Statik und erlangt eine Art Eigenleben in der Reflexion. In diesen Studien haben sich in den vergangenen Jahrzehnten Themen wie die Geschichte der Konsumkultur, des Sammelns und Repräsentierens oder die Geschichte wissenschaftlicher und experimenteller Praktiken entfaltet.

Um die Denkfigur der materiellen Kultur herum gruppieren sich einerseits mikrohistorische Forschungsansätze, etwa, wenn es um die materiellen und apparativen Voraussetzungen von Experimentalsystemen geht, um den Konsum kultureller Eliten oder um die Herstellungstechniken von Artefakten im vorindustriellen Zeitalter. Andererseits bieten sowohl das vieldeutige Wort „Kultur“ als auch die unterschiedlichen Ansätze der Kulturgeschichte die Möglichkeit, Elemente einer Geschichte in der longue durée zu entfalten oder räumlich weit auszugreifen. Geschichten von Genussmitteln oder Arzneiformen können leicht über mehrere Jahrtausende hinweg verfolgt werden. Die geographische Verbreitung bestimmter begehrter Handelswaren entlang von Migrations- und Handelslinien oder kriegerischen Auseinandersetzungen ist kein exklusives Phänomen moderner Gesellschaften.

Mit dem Versuch einer Verkehrung des Blicks vom Geist auf die Materie bekommen die materiellen Dinge eine eigene Würde, sie scheinen zurückzuschauen, wenn wir sie anschauen. Sie bieten einen Rahmen für wissenschaftliche Handlungen, sie ermöglichen sie oder leisten ihnen Widerstand oder beides, und sie erlangen auf diese Weise etwas Subjekthaftes.

Ich möchte im Folgenden zwei Werke genauer vorstellen, die sich mit den Verbindungen zwischen materieller Kultur und Wissenskultur beschäftigen. Der von Ursula Klein und Emma C. Spary herausgegebene Sammelband Materials and Expertise in Early Modern Europe trägt den Untertitel Between Market and Laboratory und schließt Fallstudien zwischen dem 16. und dem frühen 19. Jahrhundert ein, wobei der Schwerpunkt auf dem 18. Jahrhundert liegt. Pratik Chakrabartis Monographie Materials and Medicine. Trade, Conquest and Therapeutics in the Eighteenth Century ist auf das 18. Jahrhundert konzentriert und betrachtet mit Jamaika und der indischen Ostküste exemplarisch die Peripherie des British Empire sowie deren Beziehungen zum Zentrum. Anhand beider Werke lassen sich Tendenzen erkennen, die Sprache der Dinge in Wissenskulturen neu zu lesen und damit die Sicht auf die letzteren zu verändern. Während Chakrabarti sich auf die Besonderheiten der materia medica, also arzneilicher Substanzen, konzentriert, betrachten die Autoren in Klein und Sparys Band ein breites Spektrum verschiedenster gewerblich hergestellter, privat oder staatlich konsumierter Stoffe und bleiben im europäischen Kontext. Ich möchte im folgenden die beiden Bücher 
daraufhin untersuchen, wie die Fokussierung auf die Materien erfolgt und in welche interpretativen Kontexte diese eingebunden werden.

Materials and Expertise fokussiert nicht nur die Materien frühneuzeitlicher Technologien, sondern auch die Personen, die professionell mit ihnen umgingen. Das Titelwort „Expertise“ vermeidet einerseits den anachronistischen Begriff des Experten, zeigt andererseits aber, dass es schwierig ist, jene in den Intermundien von Berufserfahrung und akademischem oder professionellem Wissen erworbenen Kenntnisse kategorial zu fassen, deren Dynamik im Zeitalter der beginnenden Moderne die Autoren nachgehen. Die anachronistische systematische Aufteilung der Wissensgebiete in unsere heutigen Disziplinen wird somit vermieden, aber es wird auch keine zeitgenössische Wissensordnung bemüht, sondern man beugt sich über jene Gebiete gewerblicher Produktion, die gegen Ende des 18. Jahrhunderts zu einem Reservoir für die Wissenskulturen der neuen Experimentalwissenschaften des 19. Jahrhunderts, allen voran der Chemie, aber auch der Physik, Biologie und Pharmazie, wurden. Es geht zum Beispiel um Tinte und Farben für Drucke oder Stoffe, aber auch um metallische Stoffe, Keramik, Schießpulver oder Milch. Durchweg handelt es sich also um Materien, die nach heutiger Auffassung Stoffgemische sind und die nach den Maßgaben der experimentellen Naturwissenschaften aufgelöst, in ihre Bestandteile zerlegt gehören, um danach analysiert und wissenschaftlich produziert zu werden. Die drei großen Teile des Buchs - überschrieben „The Production of Materials“, „Materials in the Market Sphere“ und "State Interventions“ - werden zusammengehalten durch die allgemeine Hinführung zum Thema von Klein und Spary sowie durch Kleins zusätzliche, sehr erhellende Einleitungen zu den einzelnen Teilen.

Die Mehrzahl der überwiegend als Fallstudien angelegten Einzelbeiträge - nur Marcus Popplows Beitrag über die aufgeklärten agrarwissenschaftlichen Gesellschaften hat einen anderen Zuschnitt - behandelt alle drei durch die Sektionsüberschriften gesetzten Themen, wenn auch mit unterschiedlichem Schwerpunkt. So zeigt Ursula Klein am Beispiel der Äther, dass in die Erforschung dieser neuen Stoffklasse handwerkliches Herstellungswissen von Apothekern einging, das jedoch mit dem Vorgehen der neuen analytischen Chemie kombiniert wurde. Die verschiedenen Äther-Arten ${ }^{3}$ wurden aufgrund ihrer ähnlichen Herstellungsweisen sowie ihres Gebrauchs als Arzneimittel und Konsumgüter in dieselbe Stoffklasse eingeordnet. Klein bezeichnet diese Konstellation als „mixed technoscientific culture“ (S. 156). Handwerkliche, gewerbliche und akademische Aspekte und Akteure standen nicht im Gegensatz zueinander, sondern ergänzten und mischten sich. Im Grenzraum zwischen Konsum und experimenteller Chemie bewegten sich auch die von Spary vorgestellten Liköre als Materien, die „pleasure and tastefulness” bedeuteten (S. 228). Auch sie repräsentierten ein Herstellungswissen zwischen Pharmazie, Chemie und Konsumkultur. Dass die Likörhersteller nicht als 
Angehörige der akademischen Welt angesehen wurden und sozial noch unter den Apothekern rangierten, lag zum einen an der Hierarchie der hergebrachten Professionen, zum anderen aber daran, dass die „Limonadiers“ ein Wissen hatten, welches sich nicht so einfach in die Begriffe der aufgeklärten Chemie übersetzen ließ. Andererseits beteiligten sich auch viele Apotheker an der Herstellung destillierter und nicht destillierter Limonaden und trugen somit genauso wie die von ihnen verachteten Limonadiers zum Ausbau der Konsumkultur bei.

Auch die Schießpulverproduktion stellte in Seymour Mauskopfs Analyse des englischen Fallbeispiels ein Konglomerat aus verschiedenen Wissensformen dar; gleichzeitig hing sie stark von Märkten und politischen Ereignissen ab: Hatten die (privaten) Hersteller in Kriegszeiten zahlreiche staatliche Aufträge, so gerieten sie im Frieden schnell an den Rand der Existenzfähigkeit, blieb ihnen doch nur der private Markt, wo Schießpulver etwa als Tauschmittel im Sklavenhandel beliebt war. Nicht nur die gefürchteten Explosionen und das Bedürfnis nach verbesserter gleichbleibender Qualität des Schießpulvers, sondern auch die Sorge um ausreichende Lieferungen in Kriegszeiten bewogen die englische Regierung, eigene Fabrikationsstätten aufzubauen.

Die Ressourcen für die in den verschiedenen Beiträgen fokussierten Wissensgebiete waren polymorph: Sie stammten zu großen Teilen aus spezialisiertem handwerklichen Wissen, verbreiteten sich über die Druckereien, die es vervielfältigten und in einem oft konfliktreichen Prozess mit dem Wissen der Gelehrten amalgamierten, aber auch aus der Politik, die aus physiokratischen, militärstrategischen oder merkantilistischen Motiven heraus etwa die Reform der Pulverproduktion vorantrieb oder sich für neue Wege in der Mineralogie und die Verbesserungsvorschläge agrarwissenschaftlicher Gesellschaften interessierte.

Wenn man eine Rubrizierung der Beiträge versuchen will, dann lassen sich viele von ihnen in die Geschichte der gewerblichen Chemie einordnen, und in der Tat hätten wir ja auch erwartet, dass für die Weiterentwicklung von chemischem Wissen im späten 18. Jahrhundert ökonomische Motive maßgeblich und ökonomische Argumente zugkräftig waren. Nehmen wir vulgärmarxistisch die Ökonomie als Basis und das Wissen als Überbau, so können wir in den Fallbeispielen förmlich zusehen, wie die Materien miteinander um die Plätze an den Apparaten, Maschinen und Töpfen rangeln. Mit guten Argumenten versucht das Buch, die historische Grenze zwischen akademischer und gewerblicher Chemie - oder allgemeiner zwischen theoretischer und angewandter Wissenschaft - einzureißen, womit die Frage nach der Rolle der hier fokussierten Substanzen im Produktionsprozess von Waren und Wissen noch komplizierter wird.

Die Fallstudien zeigen verschiedene Konstellationen der von Klein eingeführten „Techno-Sciences avant la lettre“ (S. 322; Klein 2005), aber auch konfliktgeladene Übergänge zwischen dem Wissen der Praktiker und dem der 
Akademiker. Dabei ist für viele der hier beschriebenen Personen nicht ganz klar, welchem Lager sie zugehörten, zumal sie sich häufig der Wissenstechniken des je anderen Lagers bedienten. Schießpulverhersteller experimentierten methodisch mit verschiedenen Herstellungsverfahren, jedoch ohne dass ihre Experimente die der Herstellung zugrundeliegenden chemischen Prozesse erklärten. Die von Agustí Nieto-Galan in seinem Beitrag über die Färbekunst vorgestellten Akteure der Textilindustrie des späten 18. und frühen 19. Jahrhunderts führten Kontroversen über die Nützlichkeit der neuen Chemie für die Herstellung einer haltbaren Kleiderfarbe, andererseits übernahmen auch die Gegner der neuen Chemie einzelne Prüfverfahren von dieser. Eine plausible Erklärung, welche Qualität der Farbe in diesem Verfahren überhaupt geprüft wurde, hatte jedoch keine der Seiten. Verfahren setzten sich vielmehr durch, wenn sie handhabbar und wiederholbar waren und wenn sie Unterscheidungen erzeugten, die im Herstellungsprozess und in der Preisbildung der Waren Sinn ergaben.

Während beim Schießpulver die Standardisierungsversuche vom Staat und dessen Bestreben nach militärisch-technischer Optimierung abhingen, wurden Diskurse über Qualitätsunterschiede in anderen Bereichen von einem normierenden, moralisch konnotierten Ideal her geführt. Dies betrifft beispielsweise die Tinte, wo, wie Adrian Johns zeigt, sich die Diskussionen zwischen den Experten um die Farbqualität des Schwarz und um die Redlichkeit des Herstellers rankten. Denn nur dieser konnte garantieren, dass die teuren Zutaten des komplizierten und mit tacit knowledge verbundenen Prozesses nicht durch preiswertere von minderer Qualität ersetzt wurden.

Die Erfassung der multiplen Bedingungen, unter denen die komplexen Stoffe, von denen in den Beiträgen die Rede ist, sich verhielten, war den aufgeklärten wissenschaftlichen Experten selten in ausreichendem Umfang möglich. So schrieben sie die Stoffe in ihnen bekannte Horizonte ein, die jedoch nicht immer denen der Praktiker entsprachen oder entwarfen wie im Fall der Farben rein äußerliche Klassifikationen, die auch den Bedürfnissen des experimentellen Wissens nur unzureichend entsprachen.

Pratik Chakrabartis Fokus liegt auf der materia medica, also arzneilichen Substanzen im weitesten Sinn, sowie deren Geschichte im 18. Jahrhundert auf Jamaika und in Indien. Im Vorwort stellt der Autor einige liebgewordene Dogmen der postkolonialen Bewegung in Frage, so etwa, dass mit der Fokussierung auf lokale Praktiken allein eine Geschichtsschreibung entstehen könne, welche die scharfen Kanten der kolonialen Ausbeutung abschleifen würde. Im Gegenteil: „Exploitation is inherent to propinquity“ (S. 11); es ist gerade die Nähe zwischen Kolonisatoren und Kolonisierten, die imperialistische Ausbeutung möglich macht, und erst in der täglichen Interaktion mit den fremden Menschen und Dingen können sich Macht- und Gewaltverhältnisse etablieren. Was nicht bedeutet, dass dies immer so gelungen wäre wie von den Kolonisatoren geplant. 
Chakrabartis erstes Beispiel ist der Versuch, auf Jamaika ein modernes Medizinsystem nach dem Vorbild der aufgeklärten englischen Medizin zu etablieren, was komplett scheiterte. Das Bedürfnis nach einem modernen Medizinsystem mit einem funktionierenden Hospital ergab sich für die Briten durch die kriegerischen Auseinandersetzungen, zunächst mit den Spaniern und Franzosen, später auch mit den sogenannten Maroons, Sklaven, denen es gelungen war, sich zu befreien. Sie hatten sich in das Hinterland der karibischen Inseln zurückgezogen und lieferten sich von dort aus immer wieder Scharmützel mit den Briten beziehungsweise den Plantagenbesitzern.

Die Ausgangssituation war unübersichtlich, besonders was die professionelle und politische Zuordnung der Akteure anging. Britische Chirurgen hatten oft auf den Schiffen von Plünderern und Piraten ihr Handwerk gelernt und bauten sich durch ihre Tätigkeit und entsprechende Publikationen in Europa dann ein akademisches Renommee auf. Die Gegnerschaften, etwa zwischen Militär- und Piratenschiffen oder zwischen verschiedenen Kolonialmächten, erlaubten dennoch die Definition gemeinsamer Interessen, so dass etwa nach Plünderungen von Schiffen die Sammler und ihre Auftraggeber (in stillem Übereinkommen) ihre Sammlungen häufig zurück erhielten. Diese füllten nicht nur lokale Arzneipflanzengärten, sondern auch jene auf dem alten Kontinent. Von der erfolglosen Suche nach abbaubaren Metallen blieben als Rest Mineralbäder übrig, die als Heil- und Erholungsorte für Europäer beworben wurden.

War also die Akquisition heilender Ressourcen durchaus erfolgreich, so gilt dies nicht im selben Maße für die Etablierung sozialer Strukturen. Klimatische und soziale Verhältnisse verschworen sich in Kingston gegen die Erstellung eines Krankenhauses. Was in den Plänen der Akteure wie der Kern eines rationalen Gesundheitswesens nach europäischem Vorbild aussah, geriet in der Praxis angesichts der kolonialen Gewaltverhältnisse zu einer geschlossenen Institution. Das Krankenhaus musste mit hohen Mauern abgeschottet werden, damit die Insassen nicht entflohen. Durch die hohen Sterberaten und das ungeeignete Klima des Standortes konnte sich Kingston nicht etablieren, und es blieb bei den vormodernen Strukturen in Port Royal. Auch die Bäder wurden kein Abbild aufgeklärter europäischer Therapie und rentierten sich häufig nicht.

Die aufgeklärten Vorzeichen der Aneignung des fremden Kontinents und seiner materia medica verblassten so immer wieder vor dem Hintergrund der Brutalität, mit der die Arbeitskraft und das heilkundige Wissen der Sklaven angeeignet wurden. Soziale Trajektorien waren also auf eine spezifische, gewaltvolle Weise in die Trajektorien der Materien eingeschrieben. Aufgeklärte und liberale medizinische Praktiker erwarben zwar punktuell auch über Kooperationen Wissen, welches dann in die europäische Öffentlichkeit gelangte. Mit ihrem europäischen Wissen standen die Ärzte den Krankheiten der Sklaven und der eigenen Leute oft machtlos gegenüber, so dass lokale 
Heiler und deren zuvor als magisch abqualifizierte Wissensformen genutzt werden mussten, auch wenn es sich schlecht mit der aufklärerischen Ideologie vertrug.

Dieser Befund lässt sich auch für die zweite von Chakrabarti näher untersuchte Region bestätigen: die südostindische Coromandel-Küste, wo sich neben Madras als militärischem Hauptstützpunkt der englischen Siedlungen dicht nebeneinander außerdem niederländische, französische und dänische Niederlassungen befanden. Hier waren die sozialen Strukturen zwischen Kolonisten und Kolonisierten differenzierter ausgebildet, zumal die Präsenz verschiedener Kolonialmächte und die Verflechtung der Interessen zwischen der East India Company und dem Militär von Beginn des 18. Jahrhunderts an die Etablierung einer medizinischen Infrastruktur als zentral erscheinen ließ.

In Indien fand bereits im späten 17. Jahrhundert ein Austausch zwischen Experten unterschiedlicher europäischer Kulturen über die materia medica statt. Eine wichtige Rolle spielten Missionare, die teilweise zu Orientalisten wurden, und selbstverständlich immer wieder Militärärzte. Im Gegensatz zu Jamaika gelang es hier, ein System medizinischer Versorgung aufzubauen, dessen Akteure mit wechselndem Erfolg um Selbstständigkeit gegenüber den militärischen Strukturen rangen. Beide - militärische und medizinische Akteure - waren jedoch abhängig von den lokalen materiellen und personellen Gegebenheiten. So herrschte chronischer Mangel an den notwendigen arzneilichen Substanzen für eine medizinische Versorgung nach westlichem Vorbild. Die klimatischen Bedingungen und der lange Transport ruinierten die Zubereitungen oft, so dass man versuchte, sie lokal herzustellen oder gar auf indische Lieferanten zurückgriff, was die Verflechtung mit den lokalen Märkten und dem lokalen Wissen vorantrieb. Lokale Heilkundige, die zuerst in die Armee eingegliedert waren, konnten zwar nie ersetzt werden, jedoch wurden sie im Verlauf des 18. Jahrhunderts immer deutlicher in subalterne Positionen gedrängt, was keineswegs verhinderte, dass ihr Wissen eingesetzt wurde.

Im Kapitel „Materials and materia medica in India" breitet Chakrabarti das komplizierte Gewebe der Arzneipflanzen, der Botanik und der botanischen Gärten, des Kulturaustauschs und der Missionen aus. Knotenpunkte waren neben den militärischen und medizinischen Einrichtungen Missionsstationen und Handelsniederlassungen. Die Bedeutung der Gewürze als Handelsware nahm im 18. Jahrhundert im Vergleich zu Mittelalter und Früher Neuzeit zwar ab; um sie herum war aber ein Diskurs gewachsen, der auch dann nicht aufhörte zu wuchern, als militärische Projekte und die Etablierung von Plantagen bereits im Mittelpunkt der kolonialen Strategien standen. Sowohl im Hinblick auf den Austausch zwischen den europäischen Akteuren (Missionaren, Chirurgen und Militärs verschiedener Nationen) als auch für den historischen Ort der botanischen Kenntnisse und Sammelobjekte kann man insofern von einer histoire croisée im Sinn von Werner und Zimmermann sprechen. Missionare, 
Orientalisten und Chirurgen entwickelten ihre eigenen Netzwerke, in denen Personen, Informationen und Materien zirkulierten.

Es ist mittlerweile ein Allgemeinplatz globalhistorischer Ansätze, dass der Wissens- und Informationsfluss zwischen Europa und seinen Kolonien keineswegs nur zentrifugal war. Nicht nur die Rohstoffe, Materien, kostbare und heilende Substanzen wanderten aus der „Peripherie“ ins „Zentrum“, sondern auch das Wissen um sie, wobei die Determinanten seiner Aufnahme ${ }^{4}$ oft im „Zentrum“ blieben. So diskutiert Chakrabarti in der Einleitung die These von Kapil Raj (2006), dass indische Munshis einen wesentlichen Beitrag zur britischen Kartographie geleistet hätten. Chakrabarti hält dem entgegen, dass diese Munshis, auch wenn sie einen großen Anteil an der eigentlichen Arbeit hatten, keine wissenschaftliche Anerkennung erlangt hätten. Als Problem einer Dezentrierung in der Historiographie des Wissenstransfers bleibt die Aufgabe, die Verflechtung zwischen lokal-autochthonen und lokal-kolonialistischen Personen und Netzwerken zu beschreiben, was dann aber auch zur Folge hat, dass der zentripetale Wissensfluss eine schwer zu trennende Mischung aus Wissen-Macht-Komplexen europäischer und nicht-europäischer Materien, Personen und Strukturen ist. In diese Komplexe sind, wie auch immer gewichtet und wie auch immer von den handelnden Personen gewertet, die kolonialen (und mitunter auch die lokalen) Gewaltverhältnisse unumgänglich eingeschrieben. Chakrabartis Buch kann insofern als Plädoyer und gelungenes Beispiel für eine Re-Politisierung der Historiographie der materiellen Kultur gewertet werden.

Neben der methodischen Ähnlichkeit der Konzentration auf die Verflechtungen lokaler Praktiken mit breiter angelegten Wissenskulturen bietet die Lektüre der beiden hier besprochenen Bücher einige zufällige Übereinstimmungen: So wurde der Salpeter für die englische Schießpulverproduktion in Indien hergestellt, und die botanischen Materien aus Übersee landeten zu Zehntausenden in den europäischen botanischen Gärten. Der Aufbau eines europäisch modellierten Medizinalsystems in Indien lädt zu einem Vergleich mit den Aussagen über Expertenkulturen in Europa ein. Auch die fließenden Übergänge zwischen Wissenschaft und Kommerz waren im 18. Jahrhundert sowohl in der Karibik und in Indien als auch in Europa gleichzeitig theoretische Hindernisse und praktische Katalysatoren der Produktion von Wissen und Waren.

Werden beide Bücher unter der Fragestellung gelesen, inwiefern ihre Ansätze die Geschichte materieller Kultur weitertreiben können, dann repräsentieren sie verschiedene Strategien einer methodischen Ausweitung, nämlich einmal in den geographischen Raum (Chakrabarti) und einmal in die Tiefe der Verflechtungen zwischen Handwerk, Wissenschaft und Expertise innerhalb der kulturellen Räume Europas (Klein und Spary). Wissenstransfer kann unter verschiedenen Aspekten betrachtet werden: Soziale Differenzen (Klein und Spary) und koloniale Gewalt (Chakrabarti) sind zwei wichtige 
Perspektiven. Die Vermischung von Theorie und Praxis, von traditionellen und modernen Diskursen, die Mehrdeutigkeit des Expertentums, die Produktivität kontingenter Prozesse, aber auch das Misslingen von Herrschaftsstrategien werden in beiden Werken in bewundernswerter Tiefe und in gut lesbaren Texten exemplarisch vorgeführt.

\section{Anmerkungen}

1 „Wissensgeschichte" wird hier als übergreifender Terminus für Wissenschaftsgeschichte, Wissensgeschichte und Science and Technology Studies verwendet.

2 Einen Überblick bietet das Kompendium Kulturgeschichte von Ute Daniel von 2001.

3 Im 18. Jahrhundert wurden auch die heutigen Ester als Äther bezeichnet; „Äther“ meinte jede Verbindung einer Säure und eines Alkohols.

4. Für eine interessante Konstellation des Nicht-Transports von Wissen vergleiche Schiebinger (2004).

\section{Literatur}

Braun, Christina von, 2012. Der Preis des Geldes. Eine Kulturgeschichte. Berlin: Aufbau.

Daniel, Ute, 2001. Kompendium Kulturgeschichte. Theorien, Praxis, Schlüsselwörter. Frankfurt a. M.: Suhrkamp.

Klein, Ursula, 2005. Technoscience avant la lettre. Perspectives on Science, 13, 226-266.

Latour, Bruno, [2002] 2012. Die Hoffnung der Pandora. Untersuchungen zur Wirklichkeit der Wissenschaft. Frankfurt a. M.: Suhrkamp.

Raj, Kapil, 2006. Relocating Modern Science: Circulation and the Construction of Scientific Knowledge in South Asia and Europe. Seventeenth to Nineteenth Centuries. Houndsmill: Palgrave Macmillan.

Schiebinger, Londa L., 2004. Plants and Empire. Colonial Bioprospecting in the Atlantic World. Cambridge/MA: Harvard University Press.

Werner, Michael/Zimmermann, Bénédicte, 2002. Vergleich, Transfer, Verflechtung. Der Ansatz der "Histoire croisée" und die Herausforderung des Transnationalen. Geschichte und Gesellschaft. Zeitschrift für historische Sozialwissenschaft, 28, 607-636.

Bettina Wahrig

Abteilung für Pharmazie- und

Wissenschaftsgeschichte

Technische Universität Braunschweig

Beethovenstr. 55

38106 Braunschweig

Germany

E-Mail: b.wahrig@tu-bs.de 